psychopraxis. neuropraxis $2020 \cdot 23: 274-279$ https://doi.org/10.1007/s00739-020-00678-w Online publiziert: 24. November 2020 (c) Der/die Autor(en) 2020

\section{Peter Berger}

Klinische Abteilung für Sozialpsychiatrie, Universitätsklinik für Psychiatrie und Psychotherapie,

Medizinische Universität Wien, Wien, Österreich

\title{
Glücksspielabhängigkeit: Diagnostik und Therapie einer Verhaltensabhängigkeit
}

\section{Einleitung}

Etwa ein Prozent der Erwachsenen leidet in ihrem Leben an problematischem Glücksspielverhalten. Es entwickelt sich dabei ein immer stärkerer Drang zu spielen, dem nicht mehr widerstanden werden kann. Das Spielen beherrscht das Leben und führt in der Regel zu massiven finanziellen, familiären, psychischen oder auch rechtlichen Problemen. Es geht nicht allein darum, Geld zu gewinnen, sondern auch durch die Wirkungen des Spiels seine Befindlichkeit zu verbessern.

》) Glücksspielabhängigkeit ist oft eine schwierige diagnostische Herausforderung

Nur weniger als zehn Prozent der von der Glücksspielabhängigkeit Betroffenen suchen primär wegen dieser Erkrankung Hilfe auf. Hinter mancher schwer zu behandelnden Depression oder Angststörung kann sich eine Glücksspielabhängigkeit verbergen, die durch die enormen finanziellen und psychischen Belastungen einen wichtigen Stressfaktor darstellt. Da diese Krankheit meist verheimlicht wird, ist sie oft eine schwierige diagnostische Herausforderung. Anhand von zwei Fallberichten wird diese psychische Störung dargestellt.

\section{Fallberichte}

Fall 1. Ein 37-jähriger Patient sucht eine ambulante Therapie wegen Problemen mit Glücksspielsucht auf. Er habe mit 20 Jahren begonnen, an Glücksspielau- tomaten zu spielen. Freunde hätten ihn damals mitgenommen und das Spiel habe ihm Spaß gemacht, er habe gesehen, wie andere Geld gewonnen hätten. In den weiteren Monaten sei er auch immer häufiger alleine spielen gegangen. Er habe in dieser Zeit auch selbst einen für seine finanziellen Verhältnisse großen Gewinn gemacht, der sich in sein Gedächtnis eingeprägt habe. Er habe dann regelmäßig gespielt und es habe immer häufiger Monate gegeben, in denen er seinen gesamten Lohn verspielt hatte. Das Verlangen zu spielen, sei vor allem nach einem anstrengenden Arbeitstag aufgetreten, und er habe während des Spiels sofort „abschalten" können. Er habe in dieser Zeit aber auch anderen Interessen und immer der Arbeit nachgehen können, aber häufig sei ein starker Drang zu spielen aufgetreten, der ihn „wie magnetisch“ zum Glücksspiel gezogen hätte. Alkohol habe er während des Glücksspiels nie getrunken. Auch sonst trinke er nur selten und mäßig Alkohol.

Vor ca. drei Jahren, im Rahmen von beruflichen und persönlichen Belastungen, habe das Glücksspiel bei ihm an Intensität zugenommen. Er sei mindestens drei Mal pro Woche spielen gegangen und habe das Casino häufig erst in der Früh verlassen, typischerweise erst, als er kein Geld mehr zur Verfügung hatte. Er habe auch während der Arbeit oft ans Glücksspiel gedacht, besonders wie er Verluste durch neuerliches Glücksspiel ausgleichen könnte. Um das Spielen zu finanzieren, habe er Ersparnisse aufgelöst, das Konto überzogen und einen Kredit aufgenommen. Er habe sich „wie ferngesteuert" gefühlt und es habe ihn zum
Glücksspiel hingezogen, obwohl ihm bewusst gewesen war, dass es ihm nicht guttue. Er habe nun an Schlafstörungen und Reizbarkeit gelitten. Er sei sonst im Alltag und bei der Arbeit ein kontrollierter und besonnener Mensch. Daher sei ihm nun erstmals bewusst geworden, dass das Glücksspiel bei ihm außer Kontrolle geraten war. Er habe sich Bücher zum Thema Glücksspielabhängigkeit besorgt und gelesen und habe sich darin in seinem Problem bestätigt gefunden. In einem Casino habe er sich sperren lassen, er sei jedoch auf andere Möglichkeiten ausgewichen und sei ins nahe Ausland gefahren, um weiterspielen zu können. Vor ca. zwei Jahren habe er erstmals einen Arzt wegen Schlafstörungen aufgesucht. Dieser habe ihn auch gefragt, $\mathrm{ob}$ er besondere Belastungen habe. Er habe sich jedoch nicht getraut über seine Glücksspielabhängigkeit zu sprechen. Das verordnete Zolpidem habe er nur an wenigen Tagen genommen, da er befürchtete, dass er sonst davon abhängig werden könnte.

Im letzten Jahr, bevor er die Behandlung aufsuchte, sei sein psychischer $\mathrm{Zu}$ stand zunehmend schlechter geworden: Er habe an Ein- und Durchschlafstörungen gelitten, er habe unkontrolliert gegessen und $15 \mathrm{~kg}$ zugenommen, er habe sich bei der Arbeit immer schlechter konzentrieren können, er habe sich energielos und freudlos gefühlt, sei leicht reizbar gewesen und er habe täglich ans Glücksspiel gedacht, wobei ihn dies belastete, zeitweise seien auch Suizidideen ohne Konkretisierung aufgetreten. Bevor er sich in Behandlung begeben hatte, habe er sich gesagt: „Wenn ich jetzt noch 
einmal Spielen gehe, obwohl ich es eigentlich nicht will, dann suche ich Hilfe auf".

\section{》) Häufige Probleme sind Lügen und Verheimlichung}

Bei Behandlungsbeginn war der Patient deutlich depressiv verstimmt mit der oben erwähnten Symptomatik. Zusätzlich litt er auch an mehrfach während des Tages auftretenden, ca. eine Minute dauernden Vorstellungskonkretisierungen, in denen er Glücksspielerinnerungen wie Flashbacks sehr plastisch wiedererlebte. Diese Episoden erlebte der Patient als sehr unangenehm. Bereits eine Woche vor Behandlungsbeginn, seit dem Entschluss, konkret Hilfe aufzusuchen, war der Patient glücksspielabstinent. Die medikamentöse Behandlung erfolgte mit Trazodon abends, da die depressive Symptomatik mit ausgeprägten Schlafstörungen im Vordergrund stand. Acht Wochen nach Behandlungsbeginn war der Patient bezüglich der depressiven
Symptomatik und der Schlafstörungen deutlich gebessert und weiter spielabstinent. Auch die Flashbacks traten deutlich seltener auf und waren weniger angstbesetzt. Im weiteren Verlauf hatte der Patient zwei Rückfälle von ca. einer Woche Dauer. Er konnte sich mit den Auslösern dieser Rückfälle auseinandersetzen und war dadurch bestärkt, längerfristig Psychotherapie fortzusetzen.

Fall 2. Eine 27-jährige Frau habe vor etwa sechs Jahren im Internet an Glücksspielseiten zu spielen begonnen. Es habe ihr gefallen und sie habe jedoch nur gelegentlich gespielt, meist wenn ihr langweilig war. Vor fünf Jahren sei sie an ihren jetzigen Wohnort gezogen. In der ersten Zeit sei es ihr gut gegangen, dann habe sie sich aber zunehmend einsam gefühlt. Sie habe gearbeitet und in ihrer Freizeit immer häufiger im Internet gespielt. Das Glücksspiel habe sie entspannt und sehr gut von der Einsamkeit und Leere abgelenkt.

Seit der Kindheit leide sie an hypochondrischen Ängsten und Angst- attacken. Sie habe sich deshalb einmal kurzfristig in Behandlung begeben. Beim Glücksspiel konnte sie ihre Ängste vergessen und sie habe sich davon zumindest kurzfristig befreit gefühlt. Sie habe nie Drogen genommen und Alkohol trinke sie nur selten. Vor ca. drei Jahren habe sie einen relativ großen Gewinn gemacht. Dies und Probleme in ihrer Beziehung hätten ihre Spielfrequenz verstärkt. Sie hätte nun häufig einen intensiven und unwiderstehlichen Drang zu spielen verspürt und fast täglich im Internet gespielt, sodass sie in mehreren Monaten immer wieder ihren gesamten Lohn verspielt hatte. Sie musste sich daher Geld leihen, um ihre Lebenshaltungskosten zu bezahlen. Außerdem zog sie sich immer mehr zurück, was ihre Partnerschaft belastete. Als sie nun im letzten Monat wieder ihren gesamten Lohn verspielt hatte, drängte sie ihr Partner, sich in Behandlung zu begeben, was sie auch tat.

Im Erstgespräch war sie sehr froh, erstmals über ihre Problematik sprechen zu können. Die Einnahme von Medikamen-

Hier steht eine Anzeige. 
ten lehnte sie ab. Ab dem Entschluss, sich in Behandlung zu begeben, blieb die Patientin glücksspielabstinent. Sie berichtete in den ersten zwei Monaten der Abstinenz über einen gelegentlichen Drang zu spielen, den sie aber kontrollieren konnte, und über wiederkehrende intensive Träume vom Glücksspiel.

\section{Diskussion}

Das Bedürfnis nach Glücksspielen ist in Ländern, in denen das Glücksspiel verfügbar ist, weitverbreitet. In Österreich nimmt einer rezenten Untersuchung zufolge etwa die Hälfte der erwachsenen Bevölkerung an Glücksspielen teil [1,2]. Bei $1,1 \%$ führt das Glücksspiel zu ausgeprägten Problemen, etwa 0,6 bis 0,7\% erfüllen ausreichend Kriterien für die Diagnose einer Glücksspielabhängigkeit oder „Störung durch Glücksspiel/Pathologisches Glücksspiel“, wie diese Erkrankung nach den offiziellen diagnostischen Manualen (DSM-5 [3] oder ICD-10 [4]) bezeichnet wird.

Männer sind häufiger betroffen als Frauen. Das Geschlechtsverhältnis ist etwa 3:1. Wie bei anderen Abhängigkeitserkrankungen haben Frauen offenbar eine größere Scheu, Hilfe aufzusuchen. In Behandlungseinrichtungen beträgt der Anteil der Frauen meist nur 10 bis $15 \%$.

Nachdem das Internet beim Glücksspiel und Wetten zunehmende Bedeutung hat, wird in der Diagnostik zwischen einer Online- und Offlinevariante der Diagnose unterschieden. Das Internet macht das Glücksspiel leicht und allzeit verfügbar, nicht nur über Computer, sondern auch über Smartphone. Bevorzugt werden Glücksspielarten mit hoher Ereignisfrequenz.

\section{》) Die emotionale Wirkung des Glücksspiels ist wichtiger, als Geld zu gewinnen}

Die Glücksspielabhängigkeit ist nicht allein durch häufiges Spielen gekennzeichnet, sondern durch die zunehmende gedankliche Einengung auf das Glücksspiel. Das Glücksspiel wird zum Wichtigsten im Leben, dadurch wird dafür nicht nur viel Geld ausgegeben, sondern auch viel

psychopraxis. neuropraxis $2020 \cdot 23: 274-279$ https://doi.org/10.1007/s00739-020-00678-w c) Der/die Autor(en) 2020

\section{P. Berger \\ Glücksspielabhängigkeit: Diagnostik und Therapie einer Verhaltensabhängigkeit}

\section{Zusammenfassung}

Nach jahrelangem, häufigen Glücksspielverhalten, bei dem das Glücksspiel von den Patienten positiv und als wirksames Mittel zur Angst- und Stimmungsregulation erlebt wird, kann sich eine Glücksspielabhängigkeit entwickeln. Typisch für diese erste Phase ist, dass kein ausreichendes Problembewusstsein besteht, obwohl bereits einzelne Probleme entstanden sind. Auch in der Phase der ausgeprägten Abhängigkeit entwickelt sich nur langsam Krankheitseinsicht, und die Motivation zur Behandlung ist ambivalent. Die zunehmende psychische Belastung führt dazu, dass das Glücksspiel zunehmend als negativ und belastend erlebt wird. Daraus ergeben sich oft erst die entsprechende Krankheitseinsicht und Motivation zur Behandlung. Trotzdem bleibt die Problematik schambesetzt. Daher sollte als erster Behandlungsschritt den Patienten das nötige Verständnis für ihre Erkrankung vermittelt werden.

\section{Schlüsselwörter}

Motivation · Glücksspielabhängigkeit . Pathologisches Glücksspiel · Depression · Angststörung

\section{Gambling Addiction: Diagnosis and Treatment of a Behavioral Addiction}

\section{Abstract}

Gambling addiction can develop after many years of frequent gambling behavior in which gambling is experienced by the patient as a positive and effective means of anxiety and mood regulation. Typical of this initial phase is that there is insufficient awareness of the problem, although some problems have already developed. Even during the phase of advanced dependency, insight into the disease develops only slowly and the motivation for treatment is ambivalent. The increasing mental stress then leads to the fact that gambling is increasingly experienced as negative and burdensome. This is often the only way to gain appropriate insight into the disease and motivation for treatment. Nevertheless, the problem remains surrounded by shame. Therefore, the first treatment step should be to provide the patient with the necessary understanding of the disease.

\section{Keywords}

Motivation - Gambling addiction · Pathological gambling · Depression · Anxiety disorder
Zeit damit verbracht. Zeit, die dem Familienleben, anderen Interessen, der Arbeit oder auch sozialen Beziehungen verloren geht.

Die Betroffenen erleben eine plötzlich Drang zu spielen, der scheinbar ohne Anlass auftritt und als überwältigend und unwiderstehlich erlebt wird. Sie können dann an nichts anderes mehr denken und erleben innere Unruhe (meist auch mit vegetativen Symptomen), die sich erst durch das Glücksspiel löst. Spieler fühlen sich wie in einer anderen Welt, befreit von Sorgen. Typischerweise kann das Spiel erst beendet werden, wenn kein Geld mehr verfügbar ist. Das Spiel endet bei Glücksspielsüchtigen somit fast im- mer mit Verlust, auch wenn zwischenzeitlich Gewinne aufgetreten sind. Die Verluste führen dazu, dass das Bedürfnis entsteht, verlorenes Geld demnächst wieder zurückzugewinnen, wodurch sich das Verhalten perpetuiert. Als finanzielle Folge der Spielsucht entstehen fast immer Schulden. Der finanzielle Druck trägt dazu bei, dass weiter und mit höheren Einsätzen gespielt wird, da der Gedanke auftritt, dass nur ein großer Gewinn die finanziellen Probleme lösen kann.

Sehr typisch ist auch, dass das Ausmaß des Spielverhaltens aber auch der Folgen verheimlicht werden. Einerseits wird verheimlicht und gelogen, um trotz der massiven Folgen Geld zu bekommen und 
Tab. 1 Diagnostische Kriterien der Glücksspielabhängigkeit

Internationale Klassifikation, WHO, (ICD-10: F63.0 Pathologisches Glücksspiel) [4]

Wiederholte Episoden von Glücksspiel

Diese Episoden bringen den Betroffenen keinen Gewinn, sondern werden trotz subjektivem Leidensdruck und Störung der sozialen und beruflichen Funktionsfähigkeit fortgesetzt

Intensiver Drang zu spielen, der nur schwer kontrolliert werden kann. Ist nicht in der Lage, das Glücksspiel durch Willensanstrengung zu unterbrechen

Ständige Beschäftigung mit Gedanken oder Vorstellungen vom Glücksspiel oder mit dem Umfeld des Glücksspiels

Amerikanische Klassifikation, APA, (DSM-5: Störung durch Glücksspiel) [3]

A. Andauerndes und wiederkehrendes fehlangepasstes Spielverhalten, das sich in mindestens vier der folgenden Merkmale ausdrückt:

1. Muss mit immer höheren Einsätzen spielen, um die gewünschte Erregung zu erreichen

2. Ist unruhig und gereizt beim Versuch, das Spielen einzuschränken oder aufzugeben

3. Wiederholte erfolglose Versuche, das Spielen zu kontrollieren, einzuschränken oder aufzugeben

4. Ist stark eingenommen vom Glücksspiel

5. Häufiges Glücksspielen in belastenden Gefühlszuständen

6. Kehrt nach Verlust oft am nächsten Tag zurück, um diesen auszugleichen (dem Verlust hinterherjagen)

7. Belügt andere, um das Ausmaß seiner Verstrickung in das Spielen zu vertuschen

8. Hat eine wichtige Beziehung, seinen Arbeitsplatz, Ausbildungs- oder Aufstiegschancen wegen des Spielens gefährdet oder verloren

9. Verlässt sich darauf, dass andere ihm Geld bereitstellen, um die durch das Spielen verursachte hoffnungslose finanzielle Situation zu überwinden

B. Kann nicht durch manische Episode erklärt werden

weiterspielen zu können oder Schuldner zu vertrösten, aber auch weil das Spielen sehr schambesetzt sein kann oder auch die (berechtigte) Angst besteht, dass andere kein Verständnis dafür haben, dass man so „verrückt" sein kann, trotz massiver Probleme weiterzuspielen. Gerade das Lügen bezüglich des Glücksspielverhaltens erleben viele Betroffene als massive Belastung und als besorgniserregende Charakterveränderung.

\section{Wirkung des Glücksspiels}

Obwohl es beim Glücksspiel um Gewinn oder Verlust von Geld geht, ist bei der Glücksspielabhängigkeit der Wunsch, Geld zu gewinnen, nur ein Aspekt des Glücksspiels. Viel stärker ist die emotionale Wirkung des Glücksspiels. Bereits der Gedanke an das Spiel bewirkt die Verbesserung der Befindlichkeit. Sorgen treten in den Hintergrund und die Erwartungshaltung (bezüglich Gewinne) wird optimistisch. Während des Spiels bewirkt die Anspannung durch die Erwartung des Spielergebnisses eine ständige Stimulierung des Belohnungs- systems. Auch in der Kasuistik berichten beide Patienten, dass sie sich wie in einer anderen Welt und frei von alläglichen Sorgen fühlten und dass dies die beste Art sei, sich zu entspannen.

Durch die finanziellen Verluste aufgrund des Kontrollverlustes folgt depressive Verstimmung, die in Zusammenwirken mit dem Wunsch, die Verluste wieder auszugleichen, zu weiterem Drang zu spielen führt. Dadurch ergibt sich eine Spirale von positiven und negativen Verstärkern und das Glücksspiel verankert sich bei der Glücksspielabhängigkeit als letztendlich dysfunktionale, aber für die Patienten unmittelbar beste und einfachste Art der emotionalen Regulation.

\section{Diagnostik und Komorbidität}

In beiden diagnostischen Systemen (ICD-10 und DSM-5) [3, 4] beschreiben die diagnostischen Kriterien neben der exzessiven Häufigkeit des Spielens die ständige gedankliche Beschäftigung mit dem Glücksspiel, den intensiven Drang zu spielen, dem nicht widerstanden werden kann, die Unfähigkeit, das Spielen selbst zu beenden, und das fortgesetzte Spielen trotz gravierender Folgen (siehe - Tab. 1). Im DSM-5 werden aber noch weitere typische Verhaltensweisen angeführt, wie das Lügen bezüglich der Häufigkeit des Glücksspiels und daraus resultierender Verluste und der Drang, Verluste durch neuerliches Spielen wieder auszugleichen. Zusätzlich gibt es Kriterien, die denen der substanzgebundenen Abhängigkeiten entsprechen, wie wiederholte erfolglose Versuche, das Spielen einzuschränken oder aufzugeben, das Spielen, um Problemen $\mathrm{zu}$ entkommen oder die Stimmung zu verbessern (Wirkung), Steigerung der Einsätze, um die gewünschte Erregung zu erreichen (Toleranzentwicklung) und Unruhe und Gereiztheit beim Versuch, das Spielen einzuschränken (Entzugssymptome). Gestützt auf Untersuchungen, die unter anderem auf gemeinsame genetische Faktoren und ähnliche Aktivierungsmuster bei Substanzabhängigkeit und Spielsucht hinweisen, ist im DSM-5 die Glücksspielabhängigkeit als „Abhängigkeitsstörung ohne Substanzbezug" verzeichnet, was auch in der zukünftigen Version der ICD (ICD-11) vorgesehen ist.

Bei beiden Patienten der Kasuistik zeigt sich, dass sich die Störung über Jahre progredient entwickelt. Häufig verstärken persönliche Krisensituationen die Spielintensität und damit die Symptomatik. Bei Frauen und Patienten, die erst im späteren Leben zu spielen begonnen haben, kommt es meist rascher zur Eskalation.

\section{》) Raschere Eskalation bei Frauen und älteren Patienten}

Die meisten Glücksspielabhängigen leiden zusätzlich an anderen psychiatrischen Erkrankungen. Das Erkennen dieser Erkrankungen ist für die Planung der weiteren Behandlung wichtig. In epidemiologischen Studien zeigt sich eine sehr hohe Komorbidität mit substanzbezogenen Abhängigkeiten, affektiven Erkrankungen, Angststörungen, Persönlichkeitsstörungen und der Aufmerksamkeitsstörung [5]. Bei Patienten, die primär wegen Spielsucht Hilfe auf- 
suchen, findet sich meist eine deutlich geringere Rate von substanzbezogenen Abhängigkeiten. Eine Depression kann sowohl primär bestehen als auch sekundär im Rahmen der zunehmenden Belastungen durch die Spielsucht auftreten. In beiden Fällen verstärkt dabei die negative Befindlichkeit den Drang zu spielen und den Wunsch, damit die Stimmung zu verbessern. Bei Patient 1 der Kasuistik finden sich in der Anamnese keine psychiatrischen Vorerkrankungen, sondern es entwickelt sich bei ihm im Rahmen der Belastungen eine ausgeprägte Depression, was ihn letztendlich zur Behandlung führte.

\section{Behandlung}

Der erste Schritt in der Behandlung ist die Klärung und Förderung der Krankheitseinsicht und der Änderungsmotivation, die meist ambivalent ist. Der Patient der Kasuistik hat sich bereits vor Behandlungsbeginn mit beiden auseinandergesetzt, was prognostisch günstig, aber sonst sehr häufig nicht der Fall ist. Weitere Schritte sind die Empfehlung von Sicherungsmaßnahmen (z. B. Spielanbietersperre, nur wenig Geld bei sich haben), die Schuldenregulierung, die Analyse der persönlichen Funktionalität des Spielverhaltens, die Identifizierung von Auslösern für den Drang zu spielen, die Bedeutung des Spielens für die Emotionsregulation, Erkennen von dysfunktionalen Gedanken, die das Spielen verstärken (z.B. „Ich muss das verlorene Geld zurückgewinnen. Nur ein wirklich großer Gewinn kann meine finanziellen Probleme lösen, daher muss ich weiterspielen. Es gibt so etwas wie Glückssträhnen.") und letztlich der Aufbau von sozialen Beziehungen und Interessen.

\section{》) Förderung von Krank- heitseinsicht und Änderungs- motivation}

Die medikamentöse Therapie orientiert sich in erster Linie an der Behandlung einer komorbiden Störung. Kontrollierte Studien zeigen, dass Opioidantagonisten wie Naltrexon das Suchtverlangen reduzieren können [6]. Diese sind aber in dieser Indikation nicht zugelassen, sondern können nur „off label“ eingesetzt werden. Vielfach stehen jedoch Patienten einer medikamentösen Therapie ablehnend gegenüber, wie auch die Patientin in Fall 2.

\section{Hilfesuchverhalten und Verheimlichung der Glücks- spielabhängigkeit}

Auch wenn die negativen Folgen der Glücksspielabhängigkeit meist erst nach Jahren des Bestehens der Erkrankung zur Behandlung führen, haben sich gleichzeitig die verschiedenen positiv erlebten Wirkungen des Glücksspiels eingeprägt und die Problembewältigungsmechanismen der Betroffenen auf diese positiven Wirkungen des Glücksspiels eingeengt. Daher ist die Behandlungsmotivation in der Regel ambivalent und Betroffene suchen im Durchschnitt erst nach ca. acht Jahren Problemdauer erstmals Hilfe auf. Die wenigen Studien, die zum Hilfesuchverhalten von Glücksspielabhängigen vorliegen, zeigen, dass nur maximal zehn Prozent der Betroffenen wegen dieser Abhängigkeit Hilfe aufsuchen [7].

Falls Patienten den Wunsch in sich hegen, mit dem Spielen aufzuhören, besteht oft große Scham, darüber zu erzählen, oder es wird erwartet, dass kein Verständnis für diese Problematik besteht. Daher ist es eine besondere Herausforderung, Spielsucht bei Patienten zu erkennen, die nicht primär wegen dieses Leidens zur Behandlung kommen, wie dies auch beim Patienten (Fall 1) der Kasuistik der Fall war. Wie bereits erwähnt, leiden viele Glücksspielabhängige an Depressionen oder Angststörungen und suchen diesbezüglich eher Hilfe auf, oder kommen wegen eines Suizidversuches in Behandlung. Bei der Exploration von psychosozialen Belastungsfaktoren können etwa ungeklärte finanzielle Probleme einen Hinweis auf eine bestehende Spielsucht ergeben.

\section{Zusammenfassung}

Die präsentierten Fälle zeigen, dass sich nach jahrelangem gelegentlichen oder bereits problematischen Glücks- spielverhalten, bei dem das Glücksspiel vom Patienten positiv und als wirksames Mittel zur Spannungsregulation erlebt wird, eine Glücksspielabhängigkeit entwickeln kann. Typisch für diese erste Phase ist, dass kein ausreichendes Problembewusstsein besteht, obwohl bereits einzelne Probleme existieren. Auch in der Phase der ausgeprägten Abhängigkeit entwickelt sich nur langsam Krankheitseinsicht und die Motivation zur Behandlung ist ambivalent. Die zunehmende psychische Belastung führt dann dazu, dass das Glücksspiel zunehmend als Zwang und belastend erlebt wird. Daraus ergibt sich oft erst die entsprechende Krankheitseinsicht und Motivation zur Behandlung. Trotzdem bleibt die Problematik schambesetzt. Daher sollte als erster Behandlungsschritt den Patienten das nötige Verständnis für die Erkrankung vermittelt werden.

\section{Fazit für die Praxis}

- Glücksspielabhängige suchen nich nur wegen finanzieller Probleme oder auf Druck von Angehörigen Hilfe auf, sondern vor allem wegen psychischer Beschwerden. Das Glücksspiel ist dann nicht mehr Entspannung und Ablenkung von Problemen, sondern wird zur Belastung. Der zuerst lustvolle Drang wird dann immer öfter zum quälenden Zwang, was zur Krankheitseinsicht und der Motivation zur Änderung beiträgt.

- Glücksspielabhängigkeit ist sehr schambesetzt und wird daher meist verheimlicht, so auch im ärztlichen Gespräch, falls überhaupt ärztliche Hilfe aufgesucht wird. Hinter ungeklärten finanziellen Problemen kann sich eine Glücksspielabhängigkeit verbergen.

- Bei Komorbidität einer psychischen Erkrankung (wie z. B. Depression) und einer Glücksspielabhängigkeit sollte sich die Behandlung auf beide Erkrankungen richten. 


\section{Korrespondenzadresse}

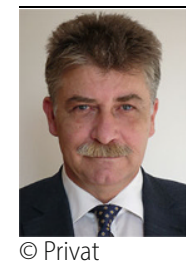

Ass. Prof. Dr. Peter Berger

Klinische Abteilung

für Sozialpsychiatrie,

Universitätsklinik

für Psychiatrie und

Psychotherapie, Medizinische Universität Wien

Währinger Gürtel 18-20,

1090 Wien, Österreich

peter.berger@

meduniwien.ac.at

Funding. Open access funding provided by Medical University of Vienna.

\section{Einhaltung ethischer Richtlinien}

Interessenkonflikt. P. Berger gibt an, dass kein

Interessenkonflikt besteht.

Für diesen Beitrag wurden vom Autor keine Studien an Menschen oder Tieren durchgeführt. Für die aufgeführten Studien gelten die jeweils dort angegebenen ethischen Richtlinien.

Open Access. Dieser Artikel wird unter der Creative Commons Namensnennung 4.0 International Lizenz veröffentlicht, welche die Nutzung, Vervielfältigung, Bearbeitung, Verbreitung und Wiedergabe in jegli- chem Medium und Format erlaubt, sofern Sie den/die ursprünglichen Autor(en) und die Quelle ordnungsgemäß nennen, einen Link zur Creative Commons Lizenz beifügen und angeben, ob Änderungen vorgenommen wurden.

Die in diesem Artikel enthaltenen Bilder und sonstiges Drittmaterial unterliegen ebenfalls der genannten Creative Commons Lizenz, sofern sich aus der Abbildungslegende nichts anderes ergibt. Sofern das betreffende Material nicht unter der genannten Creative Commons Lizenz steht und die betreffende Handlung nicht nach gesetzlichen Vorschriften erlaubt ist, ist für die oben aufgeführten Weiterverwendungen des $\mathrm{Ma}$ terials die Einwilligung des jeweiligen Rechteinhabers einzuholen.

Weitere Details zur Lizenz entnehmen Sie bitte der Lizenzinformation auf http://creativecommons.org/ licenses/by/4.0/deed.de.

\section{Literatur}

1. Kalke J, Buth S, Rosenkranz M, Schütze C, Oechsler H, Verthein U (2011) Glücksspiel und Spielerschutz in Österreich. Empirische Erkenntnisse zum Spielverhalten der Bevölkerung und zur Prävention der Glücksspielsucht. Lambertus, Freiburgi. Brsg

2. Kalke J, Wurst FM (2015) Glücksspielverhalten und Glücksspielprobleme in Österreich. Ergebnisse einer Repräsentativbefragung. Institut fü interdisziplinäre Sucht- und Drogenforschung, Hamburg
3. American Psychiatric Association (2015) Diagnostisches und Statistisches Manual Psychischer Störungen DSM-5: Deutsche Ausgabe herausgegeben von Peter Falkai und Hans-Ulrich Wittchen. Hofgrefe, Göttingen

4. Von Dilling $\mathrm{H}$ et al (Hrsg) (1994) Internationale Klassifikation psychischer Störungen: ICD-10, Kapitel V (F); Forschungskriterien/ Weltgesundheitsorganisation, 1. Aufl. Huber, Bern; Göttingen; Toronto; Seattle

5. Petry NM, Stinson FS, Grant BF (2005) Comorbidity of DSM-IV pathological gambling and other psychiatric disorders: results from the National Epidemiologic Survey on Alcohol and Related Conditions. JClin Psychiatry 66(5):564-574

6. GoslarM, LeibetsederM,Muench HM,HofmannSG, Laireiter AR (2019) Pharmacological treatments for disordered Gambling. J Gambl Stud 35:415-445

7. Slutske WS (2006) Natural recovery and treatmentseeking in pathological gambling: results of two U.S. national surveys. Am J Psychiatry 163(2):297-302

Hinweis des Verlags. Der Verlag bleibt in Hinblick auf geografische Zuordnungen und Gebietsbezeichnungen in veröffentlichten Karten und Institutsadressen neutral.

Hier steht eine Anzeige.

\section{Springer}

